\title{
Diseño de guías de laboratorio para un curso de sistemas de potencia
}

\section{Design of laboratory guides for a power systems curse}

\author{
RADA, Michell A. ${ }^{1}$ \\ RESTREPO, Diego. ${ }^{2}$ \\ LOPEZ-LEZAMA, Jesús M. ${ }^{3}$
}

\begin{abstract}
Resumen
En este artículo se presenta el desarrollo de cuatro guías de laboratorio para un curso de sistemas de potencia. Las guías incluyen los siguientes aspectos: 1) familiarizarse con el software de simulación, 2) resolver el problema de flujo de carga, 3) evaluar confiabilidad y 4) hacer compensación de potencia reactiva. En las guías se desarrollan los temas paso a paso; adicionalmente se incluyen videos para facilitar la comprensión por parte de los estudiantes.

Palabras clave: guías de laboratorio, simulación, sistemas de potencia.
\end{abstract}

\begin{abstract}
This article presents the development of four laboratory guides for a power systems course. The guides include the following aspects: 1) getting acquainted with the simulation software, 2) solving the load flow problem, 3) evaluating reliability and 4) making reactive power compensation. The guides develop the topics in a step-by-step basis; additionally, videos are included to facilitate the students understanding.
\end{abstract}

key words: laboratory guides, simulation, power systems.

\section{Introducción}

Los sistemas de potencia se encargan de transmitir grandes bloques de energía desde los centros de generación hasta los centros de consumo. Los elementos de un sistema de potencia incluyen las plantas de generación, subestaciones, líneas de transmisión y cargas. Cada uno de estos elementos se modela de forma diferente para su análisis, bien sea en estado estable o transitorio. El estudio de los sistemas de potencia incluye temas como el flujo de carga, la confiabilidad y la compensación de reactivos. En este artículo los tres temas son abordados por medio de guías de laboratorio que complementan las enseñanzas de clase y presentan los temas de forma clara, concisa y didáctica. A continuación, se describe brevemente la naturaleza de los problemas desarrollados en las guías de laboratorio.

El flujo de carga, también conocido como flujo de potencia consiste en determinar las magnitudes de las tensiones y los ángulos en todas las barras de un sistema. Estas variables son conocidas como variables de estado

\footnotetext{
${ }^{1}$ Estudiante de Ingeniería Eléctrica. Departamento de Ingeniería Eléctrica. Universidad de Antioquia, Medellín, Colombia, michell.rada@udea.edu.co

2 Estudiante de Ingeniería Eléctrica. Departamento de Ingeniería Eléctrica. Universidad de Antioquia, Medellín, Colombia, diego.restrepoo@udea.edu.co

${ }^{3}$ Ph.D., Profesor del Departamento de Ingeniería Eléctrica. Universidad de Antioquia, Medellín, Colombia, jmaria.lopez@udea.edu.co
} 
y a partir de estas se puede encontrar más información del estado estable del sistema como los flujos de potencia en líneas y transformadores y las pérdidas de potencia (Zimmerman et al., 2011). Los estudios de flujo de carga son necesarios para la gestión y planeamiento de los sistemas de potencia ya que constituyen la base de procesos más elaborados como el flujo de potencia óptimo (Biswas et al., 2018), el análisis de contingencias (Alizadeh \& Jadid, 2011) y el planeamiento de la expansión (Alizadeh-Mousavi \& Zima-Bočkarjova, 2016), entre otros.

La confiablidad de un sistema de potencia se relaciona con la capacidad de abastecer la demanda para diversas condiciones de funcionamiento, aun en situaciones donde falla uno o varios elementos. El criterio de confiabilidad más utilizado en la industria eléctrica es el $\mathrm{N}-1$. Se dice que un sistema cumple con el criterio de confiabilidad N-1 cuando puede seguir abasteciendo la demanda, incluso ante la falla de uno de sus elementos (líneas transformadores o generadores) (Cortina et al., 2017). La cuantificación de la confiabilidad se obtiene calculando índices de riesgo, el más significativo de los cuales es la energía no sumistrada. Por otro lado, los entes reguladores determinan índices globales de confiabilidad relacionados con la duración y frecuencia de las interrupciones con el objetivo de compensar clientes afectados y estimular a las empresas a prestar un mejor servicio. En la industria eléctrica existen muchos estudios que se basan en la confiabilidad. Uno de estos es el problema de interdicción que consiste en identificar los elementos que una vez fallados causan el mayor daño al sistema (Agudelo et al., 2015). A diferencia de los estudios convencionales de confiabilidad N-1 y N-2, el problema de interdicción aborda el criteiro N-k; es decir evalúa las condiciones más criticas del sistema, por ejemplo cuando ocurren ataques malintencionados (Corredor \& Ruiz, 2011).

La compensación de potencia reactiva consiste en gestionar de forma adecuada los elementos de compensación como bancos de inductores o capacitores, así como también los taps de los transformadores y los ajustes del nivel de tensión en las barras de generación. Esta gestión es indispensable para la mejora de la eficiencia energética de los sistemas de potencia. Esta se realiza tanto en sistemas de transmisión como en sistemas de distribución. Dentro de las ventajas de la compensación de reactivos se encuentran la reducción de pérdidas y el mejoramiento del perfil de tensiones. En los sistemas de suministro eléctrico esta se relaciona directamente con mejora en la calidad del servicio (Sanchez Dominguez et al., 2015). Por otro lado, la compensación de potencia reactiva es la base de estudios más detallados como el flujo de potencia óptimo reactivo (Lyu et al., 2013).

El objetivo principal de este artículo es presentar los temas mencionados anteriormente por medio de guías de laboratorio basadas en el software de simulación DIgSILENT Power Factory, las cuales fueron diseñadas para complementar los temas previamente vistos en clase.

\section{Metodología}

Para el desarrollo de las guías de laboratorio se han tenido en cuenta los temas mencionados en la sección de introducción que deberán ser vistos previamente en clase. Cada uno de estos temas requiere conocimientos de modelado de los elementos del sistema de potencia y solución de sistemas de ecuaciones no lineales. En este sentido, las guías buscan complementar los conceptos vistos en clase en lugar de reemplazarlos.

Para cada guía de laboratorio se ha diseñado un resultado de aprendizaje que consisten en enunciados acerca de lo que se espera que el estudiante sea capaz de hacer, comprender y demostrar una vez terminado un proceso de aprendizaje. Estos resultados son las habilidades medibles y los conocimientos que el estudiante adquiere o mejora en una disciplina específica. En la Tabla 1 se ilustran los resultados de aprendizaje y una breve descripción de las guías propuestas. 
Tabla 1

Resultados de aprendizaje

y descripción de las guías

\begin{tabular}{|c|c|c|c|}
\hline Guía & Tema & Resultado de aprendizaje & Descripción y observaciones \\
\hline 1 & $\begin{array}{l}\text { Introducción a DIgSILENT } \\
\text { Power Factory }\end{array}$ & $\begin{array}{l}\text { Conocer el programa de simulación } \\
\text { DIgSILENT Power Factory y } \\
\text { familiarizarse con el ingreso de los } \\
\text { elementos de un sistema de } \\
\text { potencia en su interfaz gráfica. }\end{array}$ & $\begin{array}{l}\text { - Busca familiarizar al estudiante con el } \\
\text { software de simulación. No requiere } \\
\text { conocimientos previos específicos. } \\
\text { - Puede ser desarrollada en las primera } \\
\text { semana de clases. }\end{array}$ \\
\hline 2 & Flujos de carga & $\begin{array}{l}\text { Aplicar los conceptos de flujo de } \\
\text { carga y su respectivo análisis en } \\
\text { sistemas de prueba de la literatura } \\
\text { técnica. }\end{array}$ & $\begin{array}{l}\text { - Busca aplicar el problema de flujo de } \\
\text { potencia a dos sistemas de prueba } \\
\text { conocidos en la literatura técnica. } \\
\text { - Debe ser desarrollada después de la } \\
\text { cuarta semana de clase. } \\
\text { - Se requieren conocimientos previos del } \\
\text { problema de flujo de carga. }\end{array}$ \\
\hline 3 & Confiabilidad & $\begin{array}{l}\text { Calcular el efecto de contingencias } \\
\mathrm{N}-1 \text { en un sistema de prueba } \\
\text { comparando los estados de pre y } \\
\text { post-contingencia. }\end{array}$ & $\begin{array}{l}\text { - Busca aplicar el criterio N-1 a un sistema } \\
\text { de potencia de prueba de } 6 \text { barras y } \\
\text { analizar su efecto. } \\
\text { - Debe ser desarrollada después de ver el } \\
\text { tema de flujo de potencia. }\end{array}$ \\
\hline 4 & $\begin{array}{l}\text { Compensación de } \\
\text { Reactivos }\end{array}$ & $\begin{array}{c}\text { Aplicar una de las estrategias de } \\
\text { compensación de potencia reactiva } \\
\text { vistas en clase para analizar el } \\
\text { impacto de esta en el perfil de } \\
\text { tensiones de una red. }\end{array}$ & 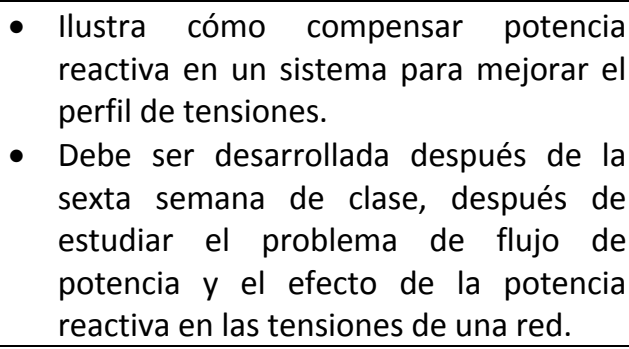 \\
\hline
\end{tabular}

Fuente: elaboración propia

\section{Resultados}

En esta sección se describe brevemente el contenido las guías desarrolladas. Las mismas se encuentran en un repositorio libre en https://mega.nz/folder/odYDwA7K\#178VSHVDyrQjGjKFExXw5w que puede ser consultado por el lector interesado. Adicionalmente, los videos de las guias 2, 3 y 4 se pueden consultar en Youtube en el siguiente enlace: https://www.youtube.com/channel/UCYen5hTRtRAT74RY_ZvfZ8g.

\subsection{Descripción general Guía 1: Familiarización con el software}

En esta primera guía se realiza la familiarización con el software DIgSILENT PowerFactory que, es una herramienta integrada para el análisis de sistemas eléctricos de potencia caracterizando técnicas flexibles de modelado y algoritmos. La versión 15.1 con la que se crearon las guías ha sido desarrollada con la nueva tecnología de programación orientada a objetos y lenguaje de programación C++. Entre las funciones principales de DIgSILENT PowerFactory, se encuentran:

- Flujos de potencia AC/DC

- Análisis de corto circuito VDE/IEC

- Análisis de estabilidad (Simulaciones RMS)

- Simulación de transitorios electromagnéticos EMT

- Análisis de contingencias

- Análisis de confiabilidad 
- Coordinación de relés de protección

- Reducción de redes

- Análisis armónico

- Cálculo de confiabilidad

- Despacho económico

- Interfaces SCADA/GIS

- Diagramas unifilares del sistema modelado

Todas estas funciones tienen acceso a una base de datos relacional común, con un sistema integrado de manejo de casos de estudio y escenarios de sistemas. En la Figura 1 se ilustran los ejemplos de aplicación predeterminados en el software DIgSILENT Power Factory, los cuales se describen brevemente a continuación.

Figura 1

Ejemplos de aplicación predeterminados

en DIgSILENT Power Factory
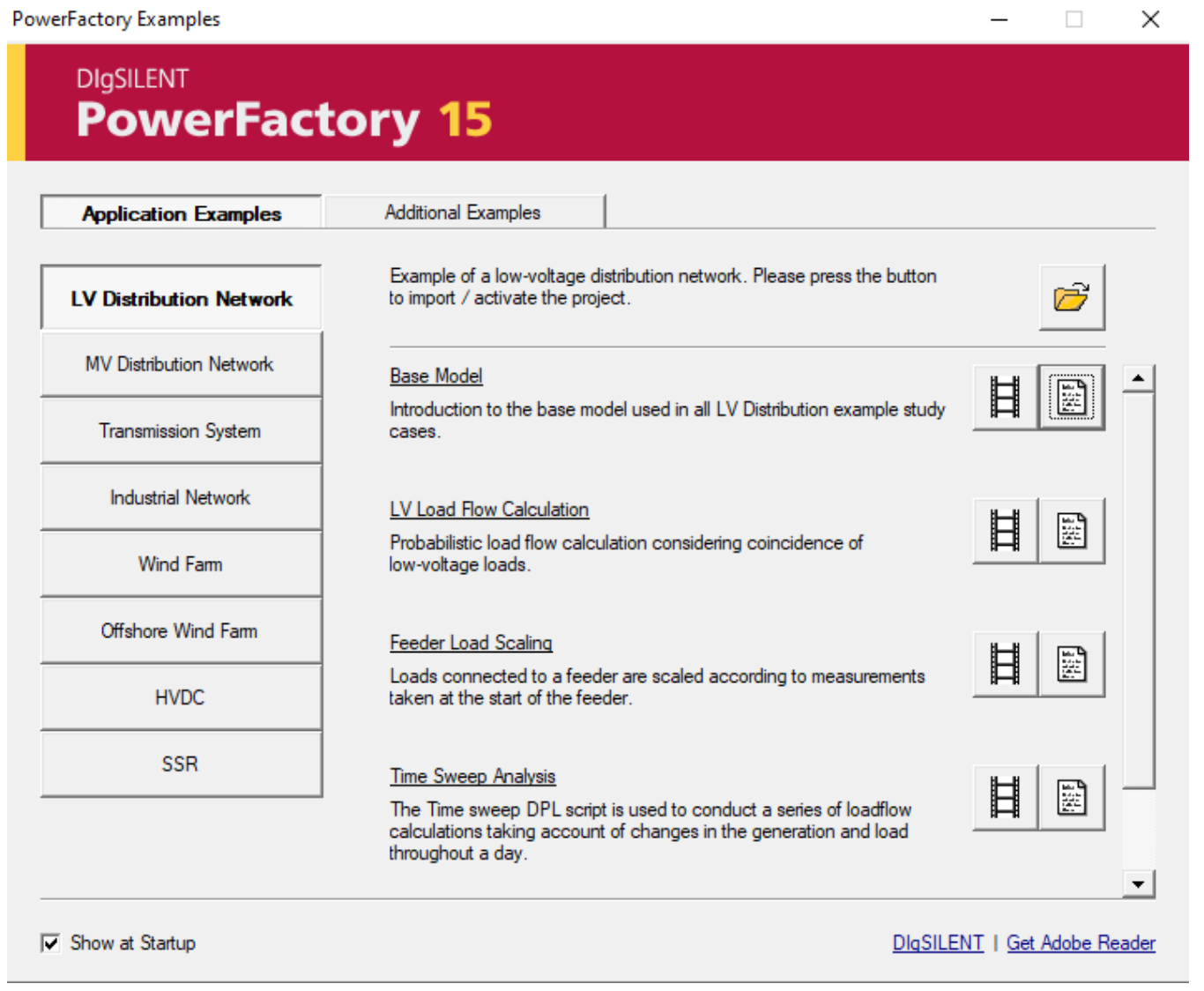

Fuente: elaboración propia

- LV Distribution Network: contiene ejemplos de distribución para tensiones menores a 11 kV. Permite ejecutar flujos de carga probabilísticos teniendo en cuenta el componente de carga fija y variable (modelo estocástico de la carga).

- MV Distribution Network: contiene ejemplos de distribución de media tensión. Permite ejecutar flujos de carga y herramientas de optimización de perfil de voltaje.

- Transmission System: Se describe un sistema de transmisión de 225/90 kV. Contiene cargas distribuidas por toda la red, subestaciones de $90 \mathrm{kV}$, centrales eléctricas, transformadores, reactores de línea y aerogeneradores. Además, contiene escenarios de cortocircuito trifásico y disparo de línea. 
- Industrial Network: Contiene el modelado de un sistema industrial con diferentes niveles de tensión y cargas de tipo AC y DC. Además, permite realizar cálculos de flujo de carga, cortocircuito, armónicos, arranque de motores, coordinación de protecciones y cálculo de riesgo eléctrico.

- Wind Farm: Se describe el modelo de un parque eólico de 6 turbinas conectado a una red de distribución de 20 kV. Contiene además modelos de VSC (convertidor de fuente de voltaje - Static Var Compensator por sus siglas en inglés), análisis de cortocircuito, PCC (potencia activa en el punto de acoplamiento común), análisis de pérdidas, factor de potencia y potencia reactiva.

- Offshore Wind Farm: Contiene el modelado de un enlace HVDC para un parque eólico marino de 400MW que consiste en turbinas eólicas. Además de análisis de convertidores de fuente de voltaje (VSC) y flujos de carga.

- HVDC: Contiene el modelado de sistemas de transmisión de corriente continua de alto voltaje (HVDC). Además de mostrar las funcionalidades del software, en la guía 1 también se hace una descripción de los elementos de la barra de herramientas principal que proporciona un acceso rápido y directo a los comandos principales del programa, así como el Data Manager que permite la administración de multiples usuarios y proyectos. Las funciones del Data Manager se ilustran en la Figura 2.

Figura 2

Funciones de la herramienta Data

Manager de DIgSILENT Power Factory

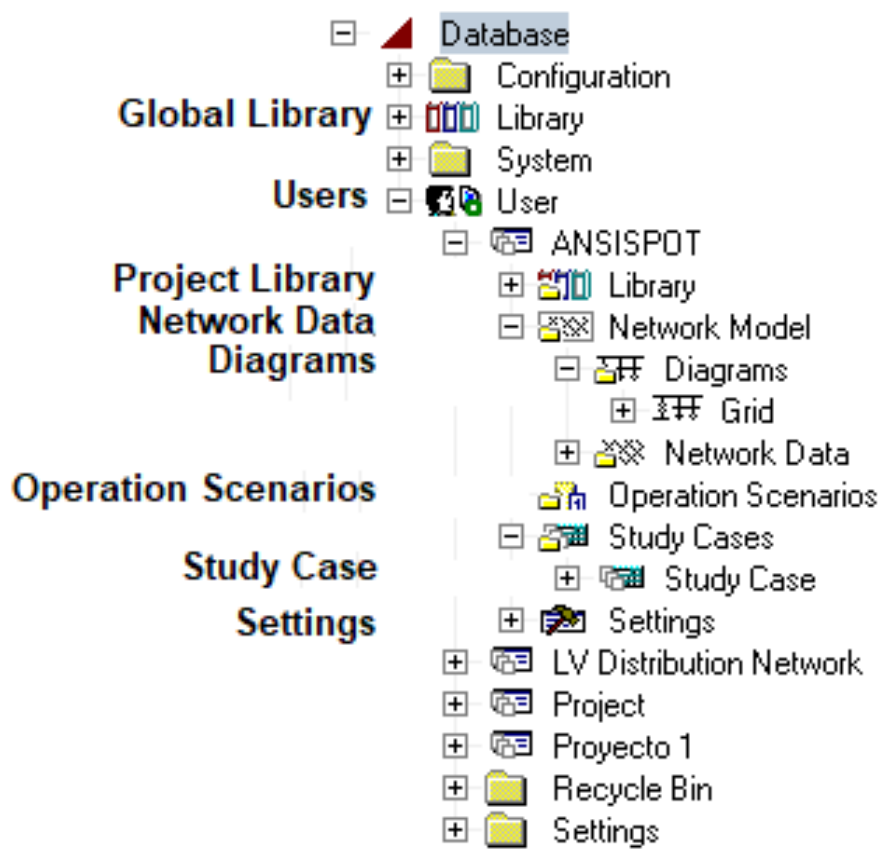

Fuente: elaboración propia

Una vez familiarizados con el entorno del sofwate la guía ilustra la forma en que se ingresan los diferentes elementos de un sistema de potencia en la interfaz gráfica. Estos incluyen barras, líneas, generadores y transformadores con sus respectivos parámetros.

\subsection{Descripción general Guía 2: Cálculo de flujos de potencia}

Resolver un flujo de potencia consiste en conocer las variables de estado (magnitudes de tensiones y ángulos nodales) para posteriormente calcular pérdidas, inyecciones de potencia y flujo de cargas en las líneas y transformadores. Existen diferentes métodos para solucionar el problema de flujo de carga, el más utilizado es el método de Newton que permite la solución de un sistema de ecuaciones no lineales. Los detalles de 
modelamiento de elementos y planteamiento de ecuaciones del flujo de potencia están por fuera del alcance de este documento y corresponde a la información que debe suministrar previamente el docente en el desarrollo del curso. En la Guía 2 se presenta un procediminento paso a paso para calcular un flujo de carga en dos sistemas de prueba ampliamente utilizados en la literatura técnica: el sistema de Wood and Wollenberg de 6 barras y en el sistema IEEE de 9 barras. A continuación, se describe brevemente el contenido de la Guía 2.

Inicialmente, se presenta el sistema de prueba y se reportan sus parámetros. Los datos de los sistemas de prueba se pueden consultrar en los archivos de Matpower (Zimmerman et al., 2011) y en un repositorio de casos de prueba disponible en (Washington, 2018). Luego, se indica cómo crear un nuevo proyecto. En la Figura 3 se ilustra el espacio de trabajo global del DIgSILENT Power Factory.

Figura 3

Espacio de trabajo del DIgSILENT Power Factory

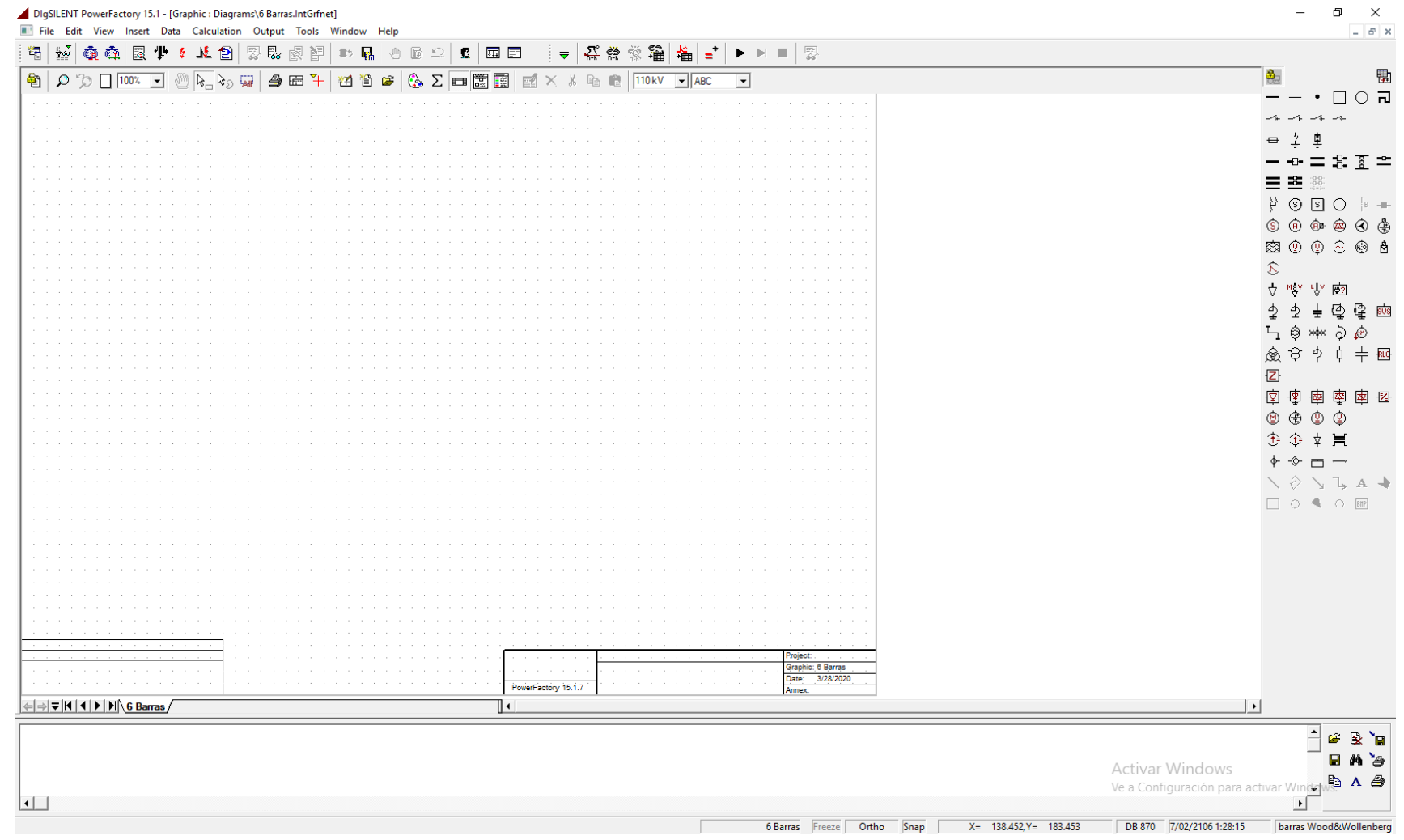

Fuente: elaboración propia

Para empezar a añadir elementos y crear el sistema de potencia, es importante saber que se deben agregar primero los buses entre los que se conectará cada elemento ya que para el caso de este software no se pueden agregar los elementos sin antes tener los extremos (buses) a los cuales estarán conectados. La guía indica entonces cómo ingresar los buses y posteriormente los elementos que conectan los buses (líneas y transformadores). Finalmente, se pueden ingresar las cargas y los generadores. A manera de ilustración, en la Figura 4 se presenta el cuadro de diálogo para ingreso de generadores. Note que este generador en particular corresponde a la barra de referencia. Para este generador no es necesario especificar la salida de potencia activa y reactiva, pues esta se calcula después de resolver el flujo de potencia para cumplir con los balances nodales del sistema y considerar las pérdidas. Notese además que es posible ingresar o ajustar la curva de capacidad del generador en cuestión. En la Figura 5 se presenta, a manera de ilustración, el sistema de prueba Wood and Wollenberg de 6 barras tal como quedaría en el espacio de trabajo del DIgSILENT Power Factory. 
Figura 4

llustración el cuadro de diálogo

para información de generadores

Synchronous Machine - 6 Barras\G1.ElmSym *

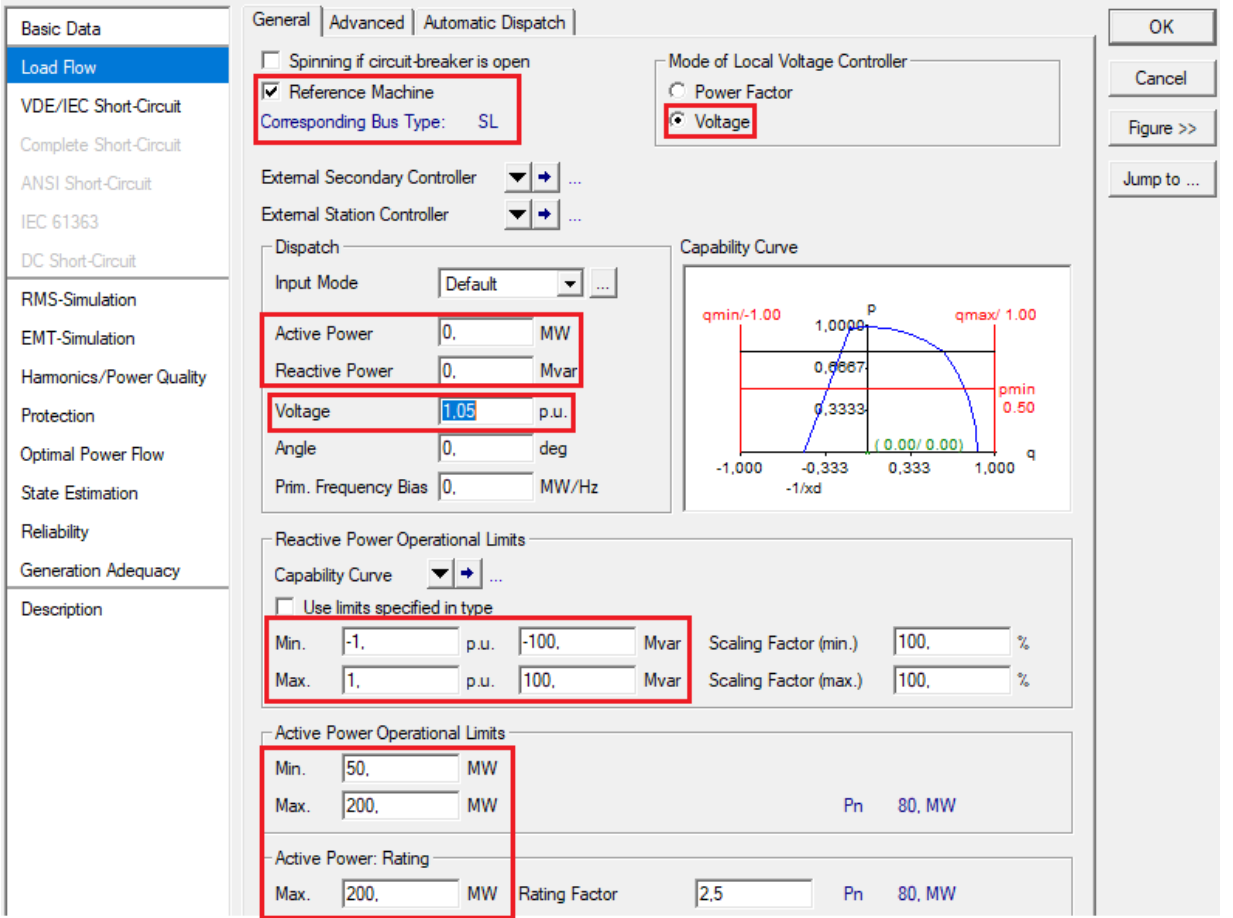

Fuente: elaboración propia

Figura 5

Sistema de 6 buses de Wood and Wollenberg modelado en DIgSILENT

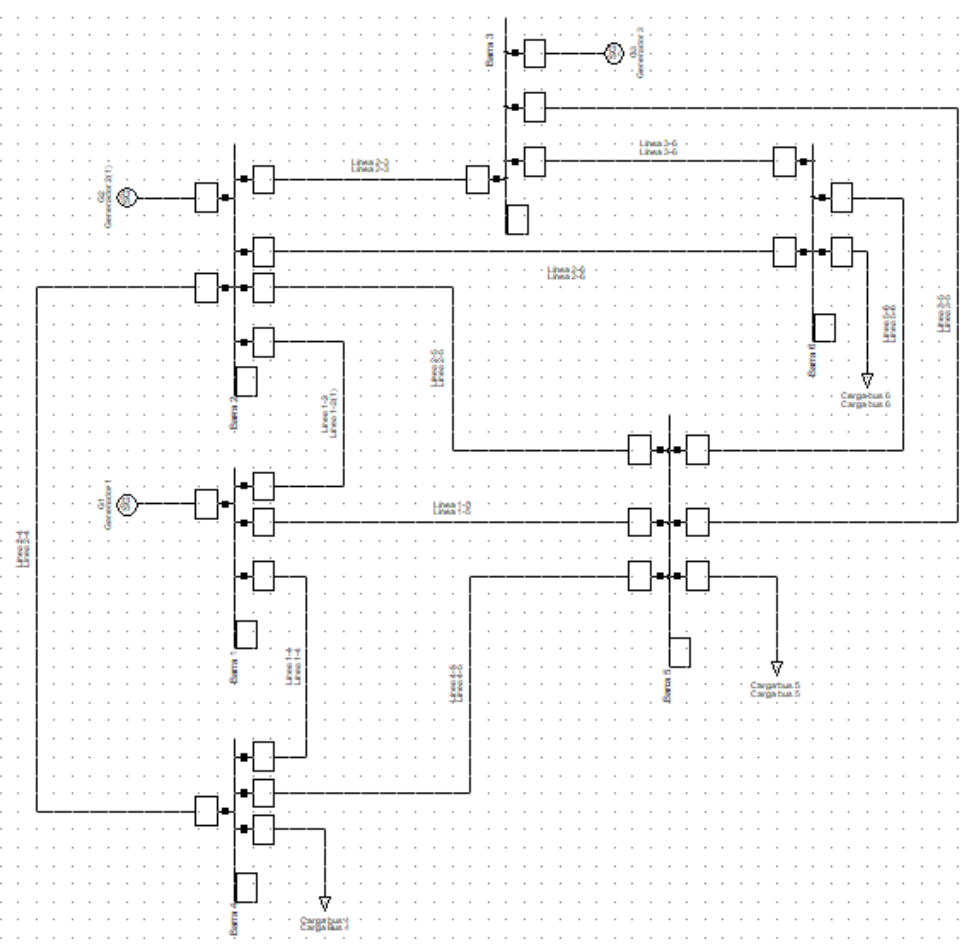

Fuente: elaboración propia 
Una vez se tiene el sistema montado en el espacio de trabajo se puede correr el flujo de carga usando el ícono correspondiente en la barra de herramientas del software. Cuando se tiene los resultados gráficos del flujo de carga, se debe tener en cuenta la convención de colores para identificar elementos problemáticos. En la Figura 6 se ilustra esta convención de colores. Finalmente, en la Figura 7 se observa el detalle de los resultados de flujo de carga y la información suministrada por el software para la línea 1-4 del sistema de Wood and Wollenberg.

Figura 6

Convención de colores según

cargabilidad de los elementos

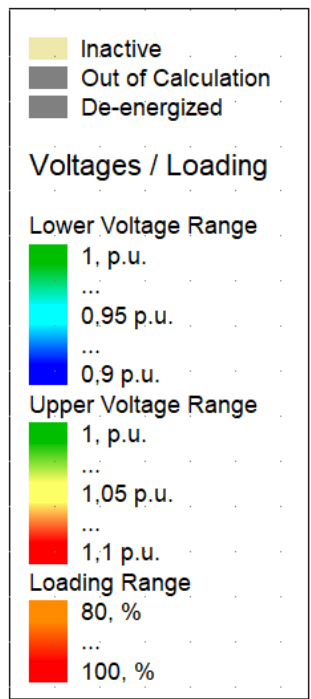

Fuente: elaboración propia

Figura 7

Detalle de los resultados del

flujo de carga en la línea 1-4

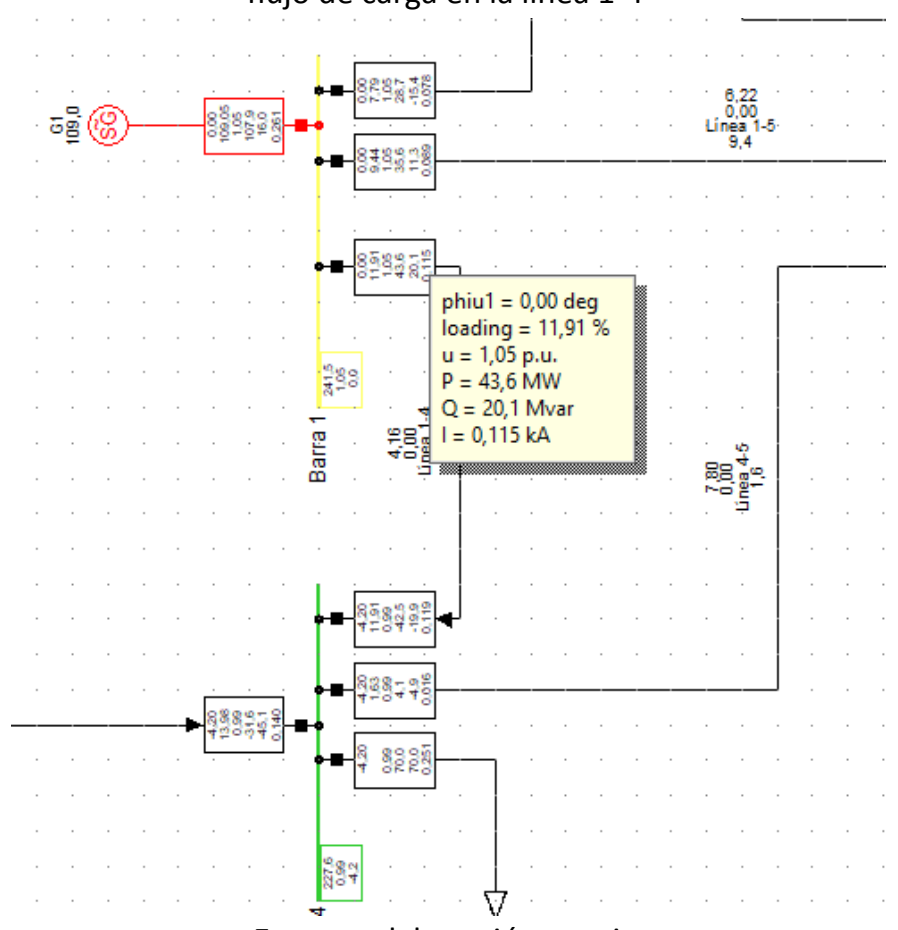

Fuente: elaboración propia 


\subsection{Descripción general Guía 3: Análisis de confiabilidad}

En esta guía se utiliza el sistema de Wood and Wollenberg previamente analizado en la Guía 2. Inicialmente, se debe buscar el caso y activarlo. Una vez activo el proyecto se debe calcular nuevamente un flujo de carga como se indicó en la Guía 2. Esto se hace con el propósito de comparar el comportamiento del sistema eléctrico de potencia en condiciones normales de operación con su comportamiento en diferentes casos de contingencias $\mathrm{N}$ 1.

Una vez ejecutado el flujo de carga del sistema, se procede a activar las herramientas para el análisis de contingencias que posee el software. Para esto se busca en la barra de herramientas la opción 'Change Toolbox'. Al dar click en esta opción se desplegará una lista de opciones, en la que se debe seleccionar 'Contingency Analysis' como se muestra en la Figura 8.

\section{Figura 8}

Lista de opciones de la herramienta

Change Toolbox

\begin{tabular}{|c|c|}
\hline $\bar{\nabla}$ & 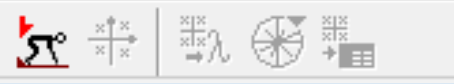 \\
\hline & Contingency Analysis \\
\hline & Quasi-Dynamic Simulation \\
\hline & RMS/EMT Simulation \\
\hline$\checkmark$ & Modal Analysis \\
\hline & Harmonics/Power Quality \\
\hline & Reliability Analysis \\
\hline & Optimal Power Restoration \\
\hline & Generation Adequacy Analysis \\
\hline & Distribution Network Tools \\
\hline & Optimal Capacitor Placement \\
\hline & Protection \\
\hline & Additional Tools \\
\hline & User-defined Tools \\
\hline
\end{tabular}

Fuente: elaboración propia

Una vez seleccionada la opción 'Contingency Analysis', se habilitará en la barra de herramientas diferentes opciones para el análisis de contingencias. Entre las opciones se tiene:

- Contingency Definition: Esta opción proporciona un método fácil para que el usuario genere casos de falla o contingencias.

- Contingency Analysis: Esta opción permite configurar según sea necesario y ejecutar la contingencia que se desee.

- Contingency Comparison: Esta opción proporciona una herramienta que le permite al usuario comparar los resultados de diferentes cálculos de contingencias.

- Show Contingencies: Esta opción permite visualizar la lista de contingencias que el usuario tiene a disposición.

- Show Fault Cases/Groups: Esta opción da acceso a la carpeta de fallas de la biblioteca interna del software. 
- Report Contingency Analysis Results: Esta opción brinda la posibilidad al usuario de generar un reporte de resultados de diferentes contingencias.

En este caso se selecciona la opción 'Contingency Definition'. Al seleccionar esta opción se ejecuta una ventana emergente. De esta ventana se seleciona la opción 'Generate Contingency Cases for Analysis'. Así mismo, en la sección 'Outage Level' se habilita la opción ' $n-1$ cases' que corresponde al evento en el cual se presenta la salida de un elemento del sistema a la vez. Para finalizar, en la sección de 'Network Components' se habilitan los componentes que el usuario desee incluir en los casos de contingencias. Una vez caracterizadas los casos de contingencia a realizar, se da click en la opción 'Execute' ubicada en la esquina superior derecha de la ventana emergente como se ilustra en la Figura 9.

Figura 9

Lista de opciones de la herramienta Change Toolbox

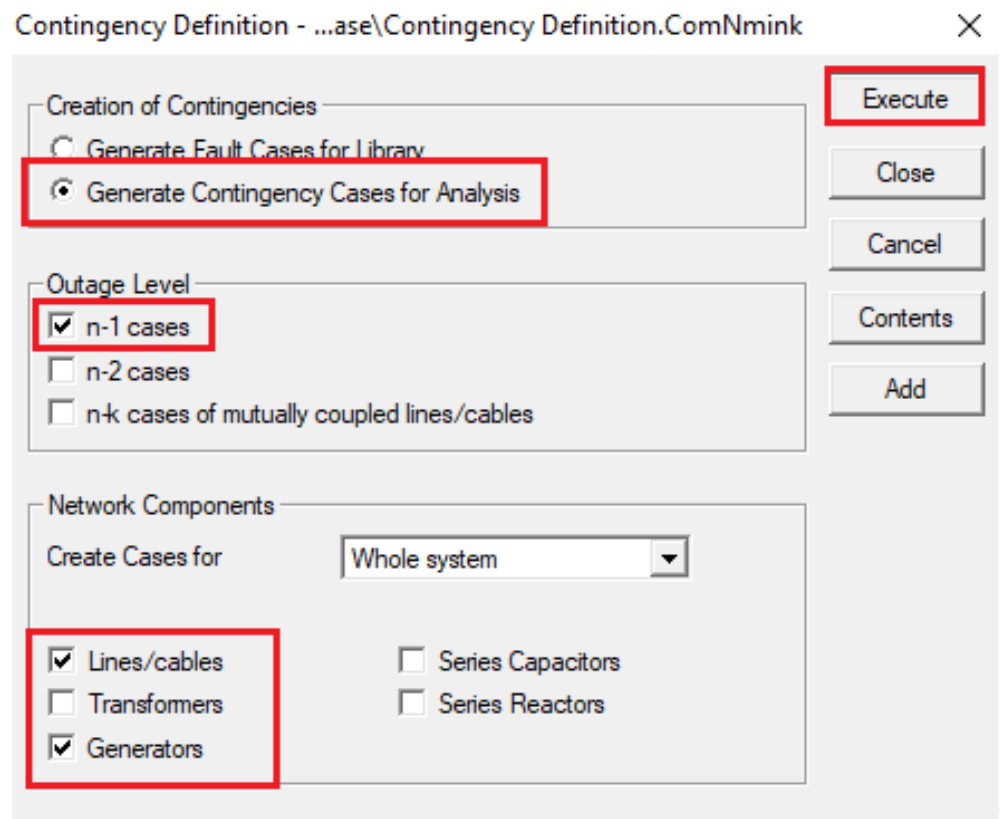

Fuente: elaboración propia

Una vez ejecutado los casos de contingencias posibles, se puede corroborar la ejecución de cada uno de estos, a través de una ventana de resultados ubicada en la parte inferior del área de trabajo. Si se desea realizar el análisis y observar el comportamiento del sistema eléctrico de potencia ante una contingencia $\mathrm{N}$ 1 en particular, en la barra de herramientas se selecciona la opción 'Show Contingencies'. Una vez seleccionada esta opción, se ejecutará una ventana emergente como se muestra en la Figura 10. En esta, se puede observar la lista de cada uno de los posibles casos de contingencia. 
Figura 10

Ventana emergente de la herramienta

'Show Contingencies'

Contingency Case - Study Cases\Study Case \Contingency Analysis :

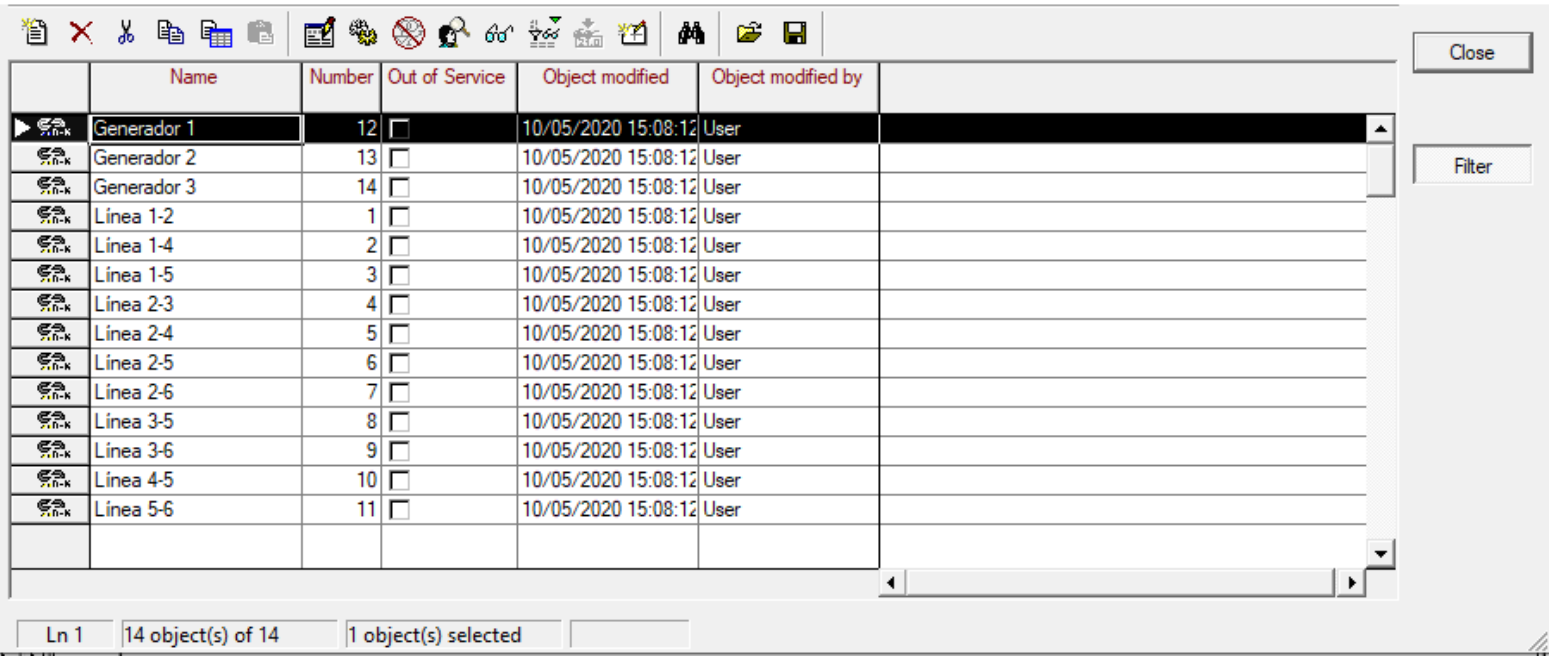

Fuente: elaboración propia

Si se desea por ejemplo analizar el comportamiento del sistema ante la salida de una la línea de transmisión de nombre en particular, es necesario ubicarse sobre el elemento que se desea analizar y dar doble click. En ese momento se ejecutará una ventana emergente como se ilustra en la Figura 11, donde se ha seleccionado la contingencia de la línea 1-5. En esa ventana, se debe dar click en la opción 'Execute' ubicada en la esquina superior derecha. Una vez ejecutado este caso de contingencia, en la ventana de trabajo se podrá observar el comportamiento del sistema de potencia en contingencia N-1. Es decir, la línea de transmisión etiquetada como 'Línea 1-5' estará fuera de servicio.

Figura 11

Ventana emergente para análisis de una contingenia en particular

Contingency Case - Study Cases \Study Case \Contingency Analysis \Línea 1-5.ComOutage

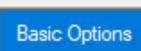

General | Advanced |

Name Linea 1-5

$\Gamma$ Not analysed

Fault Case $\quad \boldsymbol{\nabla} \rightarrow$.

Number 3

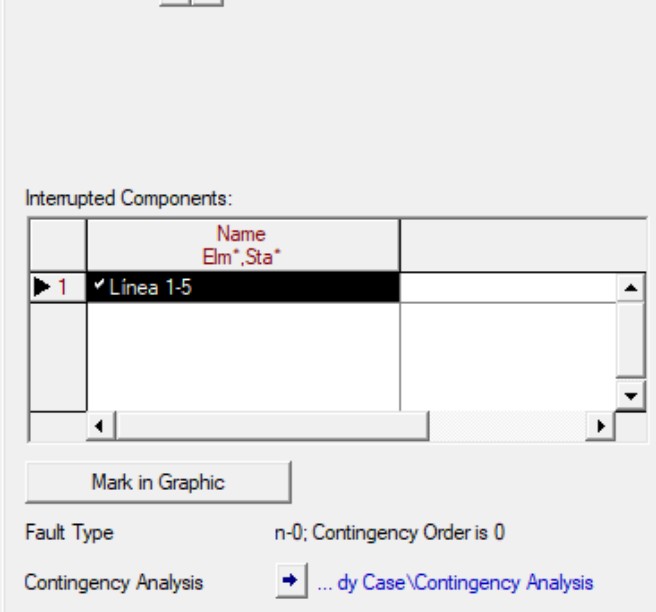

Fuente: elaboración propia 
Una vez ejecutada la contingencia se puede analizar el comportamiento del sistema de potencia. Por ejemplo, se desea observar el porcentaje de aumento de la cargabilidad de las líneas asociadas al 'Nodo 1'. Para esto, se comparan los resultados arrojados por el flujo de carga con todos los elementos del sistema en funcionamiento y para ese caso particular de contingencia.

En la Figura 12, se observa en los recuadros rojos el porcentaje de cargabilidad de las líneas de transmisión etiquetadas como 'Línea 1-2' y 'Línea 1-4' cuando el sistema está en operación normal (sin contingencia). Se puede observar que la 'Línea 1-2' tiene una cargabilidad de 7.8\% mientras que la cargabilidad de la 'Línea 1-4' es de $11.9 \%$. Por otro lado, en la Figura 13 se puede observar el porcentaje de cargabilidad de las líneas de transmisión 'Línea 1-2’ y 'Línea 1-4’ cargadas al 13.3\% y 15.7\%, respectivamente. Este aumento en la cargabilidad equivale a un 5.5\% más para la 'Línea 1-2' y un aumento de 3.8\% para la 'Línea 1-4' y se debe a la contingencia de la 'Línea 1-5'. Análisis similares se pueden hacer con otras contingencias.

Figura 12

Porcentaje de cargabilidad de la Línea 1-2

y Línea 1-4 en operación normal

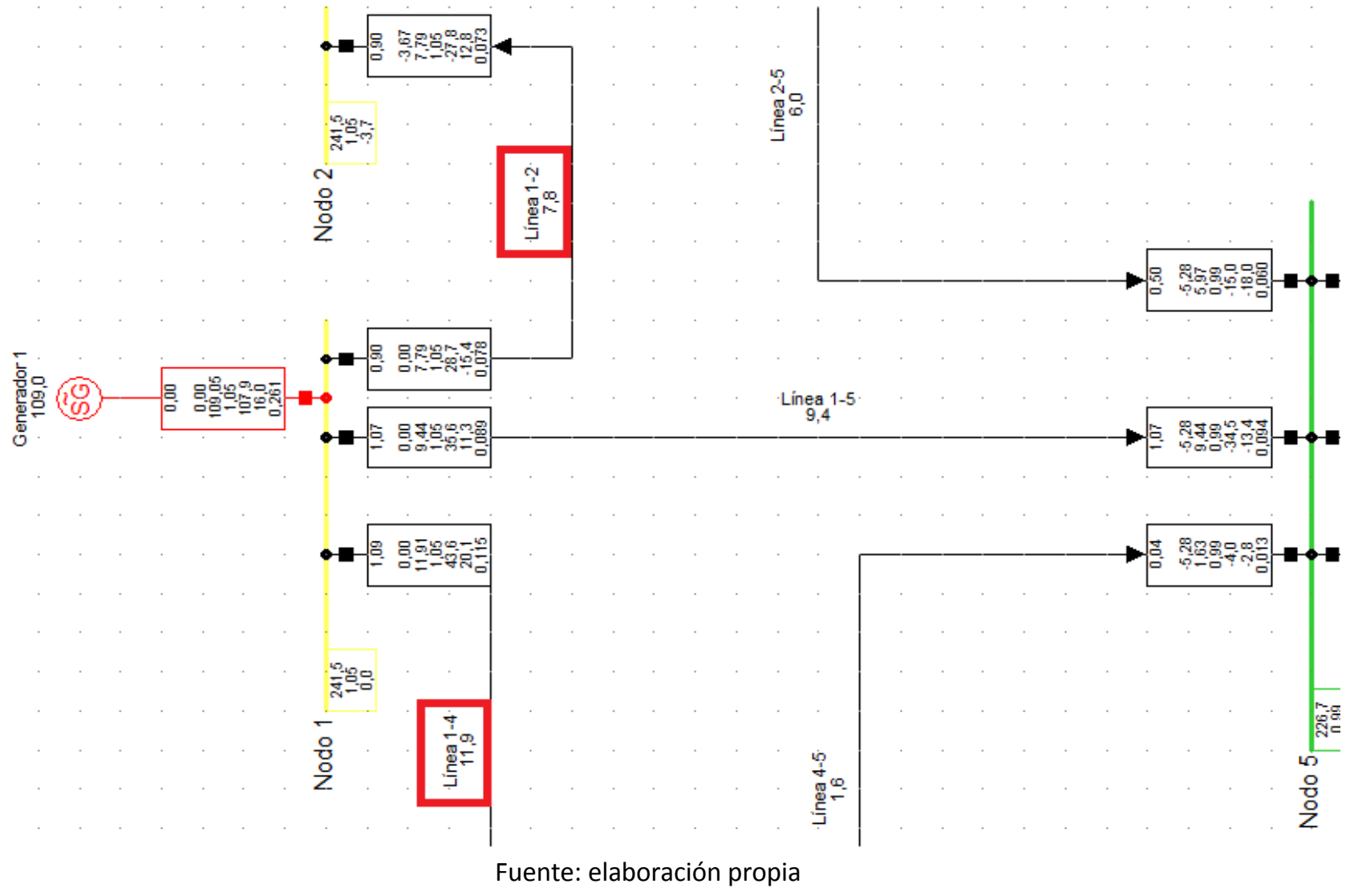


Figura 13

Porcentaje de cargabilidad de la Línea 1-2

y Línea 1-4 cuando falla la línea 1-5

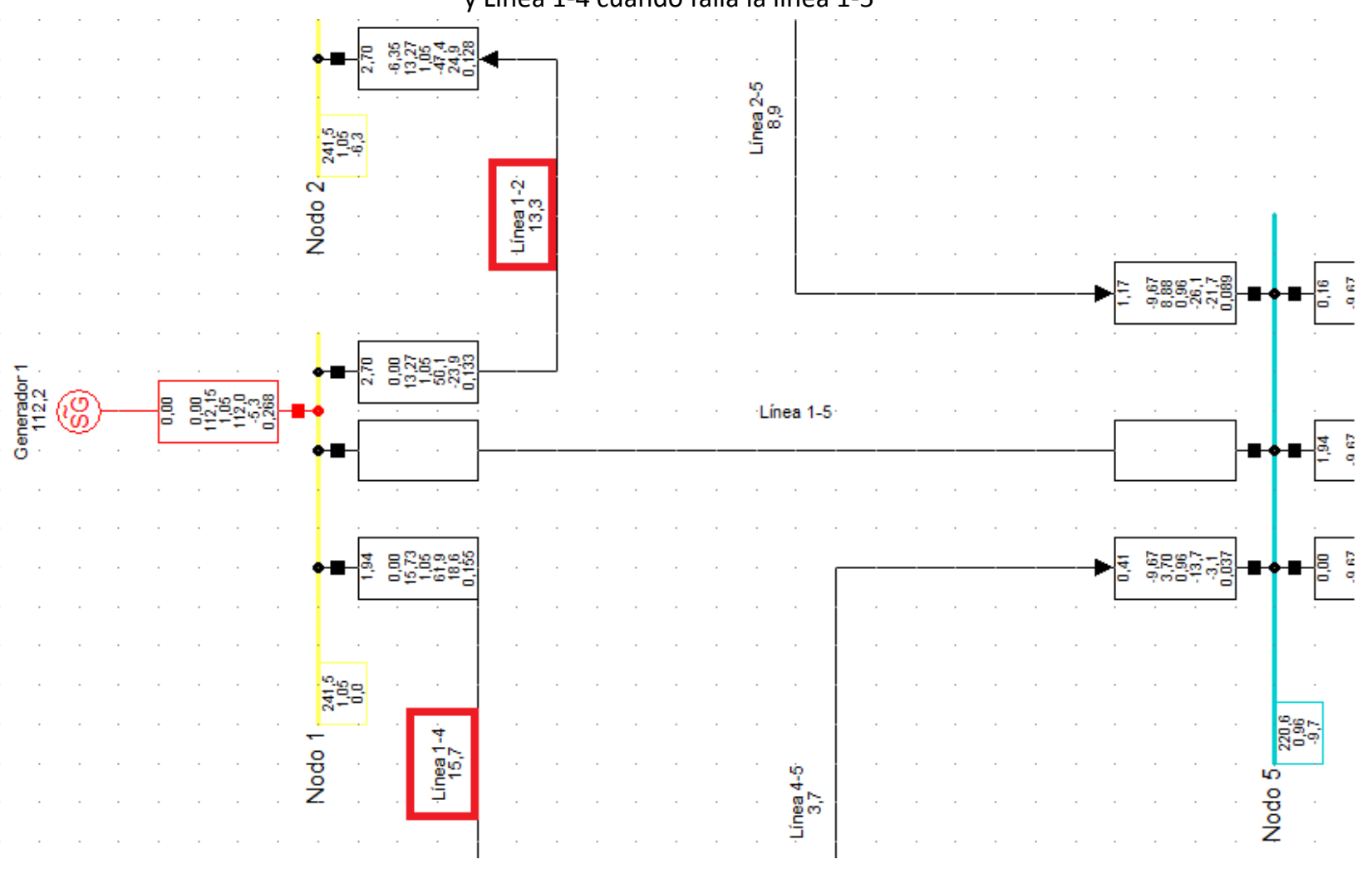

Fuente: elaboración propia

\subsection{Descripción general Guía 4: Compensación de potencia reactiva}

Tanto los elementos de un sistema de potencia como los equipos eléctricos a nivel industrial y domiciliario son diseñados para operar en ciertos niveles de tensión; sin embargo, estos tienen un margen de operación tolerable que comúnmente se encuentra entre un $-10 \%$ y $+10 \%$ de la tensión nominal. Si los equipos operan a una tensión por debajo de este margen, los equipos trabajarán con menor potencia y de una manera ineficiente; si estos operan por encima de este margen los equipos y elementos del sistema estarán sometidos a esfuerzos térmicos que terminarán afectando su aislamiento.

Por otra parte, en los sistemas AC el voltaje está estrechamente relacionado con la potencia reactiva por lo que al tener sobretensiones o caidas de tensión es importante hacer control de la potencia reactiva mediante elementos de compensación como capacitores, reactores, generadores, taps de transformadores, FACTS, etc.

En esta guía se presenta un ejemplo de compensación de potencia reactiva. Para ello, se utiliza nuevamente el sistema de 6 barras de Wood and Wollenberg. Una vez cargado el sistema se corre el flujo de carga y se verifican tensiones. En los nodos de voltaje controlado o nodo de generación el control de tensión es directo y se realiza ajustando la tensión en la ventana de diálogo de cada generador. Si se ajustan las tensiones en todos los generadores en 1.0 p.u. la tensión en la barra 4 estará por debajo de su valor nominal como se observa en la Figura 14. 
Figura 14

Tensión resultante en la barra 4 al ajustar

las tensiones de generación en 1.0 p.u.

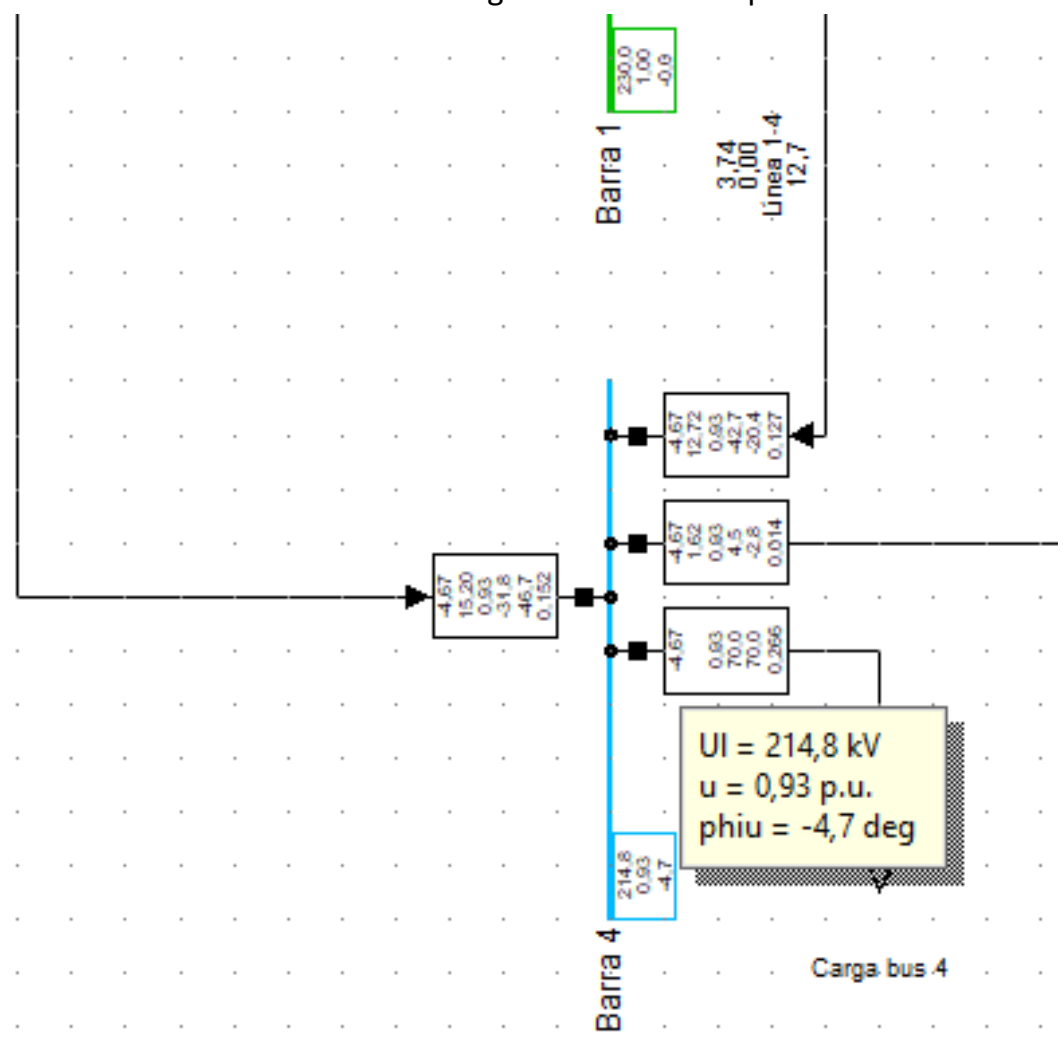

Fuente: elaboración propia

Para corregir o elevar la tensión de la Barra 4, se selecciona de la barra de elementos la opción Shunt/Filter C. Una vez se ubica el elemento en la Barra 4 como se observa en la Figura 15, se da doble click sobre el elemento para caracterizarlo. Cuando se da doble click sobre el filtro capacitivo de la Barra 4, aparecerá un recuadro. En este caso, solo se especifican las opciones de Nominal Voltage (voltaje nominal) y Rated Reactive Power, $C$ (potencia nominal reactiva). Inicialmente se modifica la tensión nominal a $230 \mathrm{kV}$ con respecto al valor predeterminado y se establece un valor de $50 \mathrm{MVar}$ con el fin de calcular nuevamente un flujo de carga y ver el efecto de la compensación en el sistema. En la figura 16, se ilustra el nuevo valor de la tensión en la nueva barra después de realizar la compensación. Se puede observar que ha pasado de 0.93 a 0.96 p.u.; sin embargo, sigue estando alejada de 1.0 p.u. que es el valor deseable. 
Figura 15

Instalación de un banco

capacitivo en la barra 4

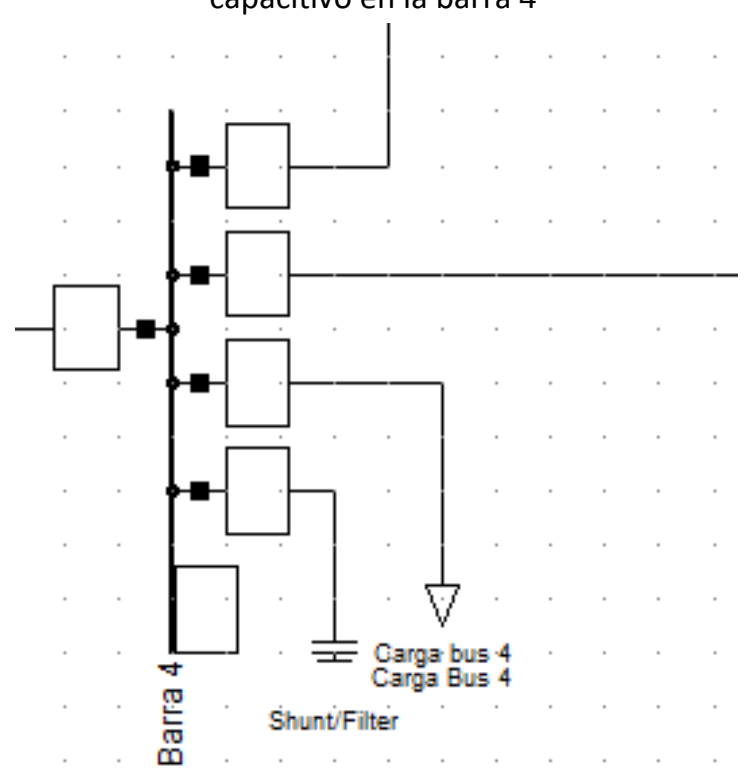

Fuente: elaboración propia

Figura 16

Tensión en la barra 4 con un banco de capacitores de 50 MVar

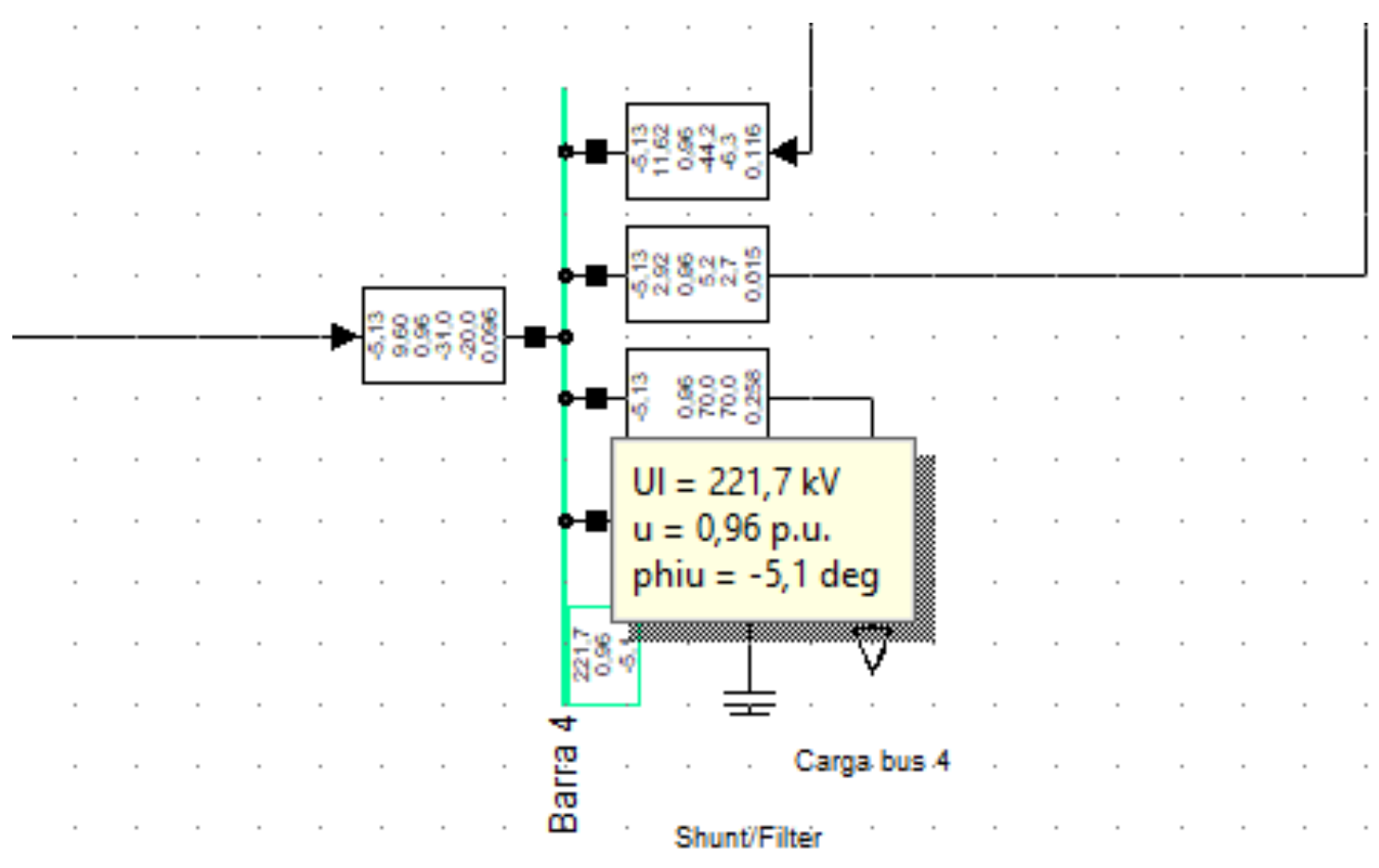

Fuente: elaboración propia

Para elevar más la tensión en la barra 4 se opta por aumentar la potencia nominal del banco capacitor a 99 MVar, obteniendo el resultado que se ilustra en la Figura 17. Note que en este caso se ha alcanzado una tensión de 1.0 
p.u. El mismo procedimiento se puede repetir para otras barras de carga del sitema. En caso de sobretensiones también se tiene la opción de bancos de reactores.

Figura 17

Tensión en la barra 4 corregida

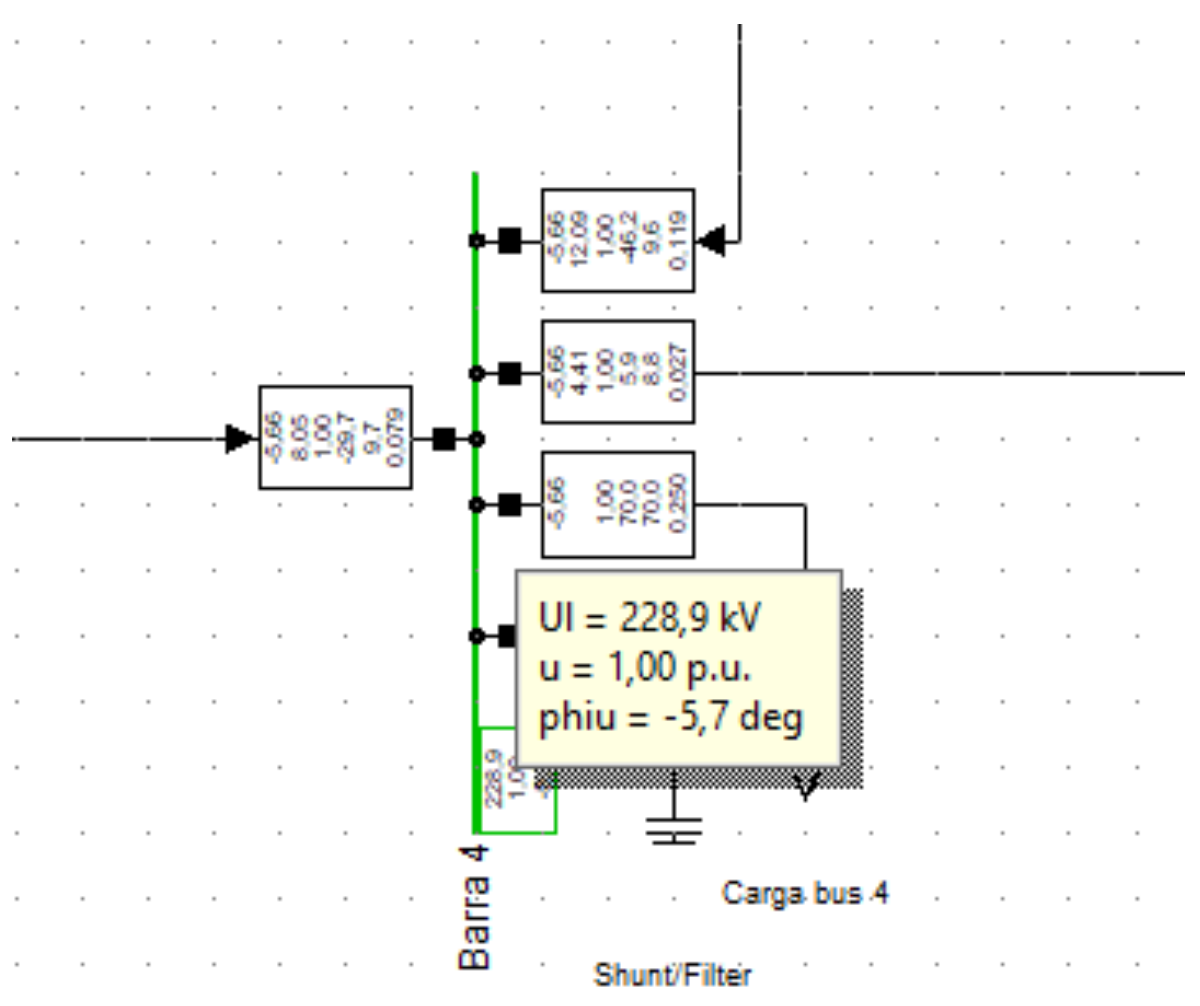

Fuente: elaboración propia

\section{Conclusiones}

En este artículo se presentó el desarrollo de cuatro guías de laboratorio diseñadas para complementar los temas de un curso de sistemas de potencia en la carrera de ingeniería eléctrica, energética y afines. Las guías desarrolladas incluyen temas como el estudio de flujos de potencia, la confiabilidad y la compensación de potencia reactiva, los cuales son fundamentales para la gestión de los sistemas eléctricos y constituyen la base de estudios más especializados. Estos temas son comunes en los cursos de sistemas de potencia y cada uno de ellos implica un desarrollo matemático complejo que debe ser visto previamente en clase. El objetivo de las guías es presentar la aplicación directa de los temas vistos en clase, usando sistemas de prueba ampliamente utilizados en la literatura técnica. Cada guía es enfocada en ayudar a alcanzar un resultado de aprendizaje específico de la materia. Adicionalmente, las guías cuentan con videos de respaldo que se encuentran disponibles en Youtube para facilitar la comprensión de los estudiantes.

\section{Referencias bibliográficas}

Agudelo, L., López-Lezama, J. M., \& Galeano, N. M. (2015). Vulnerability Assessment of Power Systems to Intentional Attacks using a Specialized Genetic Algorithm. DYNA, 82(192), 78-84.

https://doi.org/10.15446/dyna.v82n192.48578 
Alizadeh, B., \& Jadid, S. (2011). Reliability constrained coordination of generation and transmission expansion planning in power systems using mixed integer programming. Transmission Distribution IET Generation, 5(9), 948-960. https://doi.org/10.1049/iet-gtd.2011.0122

Alizadeh-Mousavi, O., \& Zima-Bočkarjova, M. (2016). Efficient Benders cuts for transmission expansion planning. Electric Power Systems Research, 131, 275-284. https://doi.org/10.1016/j.epsr.2015.10.022

Biswas, P. P., Suganthan, P. N., Mallipeddi, R., \& Amaratunga, G. A. J. (2018). Optimal power flow solutions using differential evolution algorithm integrated with effective constraint handling techniques. Engineering Applications of Artificial Intelligence, 68, 81-100. https://doi.org/10.1016/j.engappai.2017.10.019

Corredor, P. H., \& Ruiz, M. E. (2011). Against All Odds. IEEE Power and Energy Magazine, 9(2), 59-66. https://doi.org/10.1109/MPE.2011.940266

Cortina, J. J., López-Lezama, J. M., \& Muñoz-Galeano, N. (2017). Modelo de Interdicción de Sistemas de Potencia considerando el Efecto de la Respuesta a la Demanda. Información Tecnológica, 28(3), 197-208. https://doi.org/10.4067/S0718-07642017000300020

Lyu, J.-K., Heo, J.-H., Park, J.-K., \& Kang, Y.-C. (2013). Probabilistic Approach to Optimizing Active and Reactive Power Flow in Wind Farms Considering Wake Effects. Energies, 6(11), 5717-5737. https://doi.org/10.3390/en6115717

Sanchez Dominguez, J., Cerqueira Junior, A. J., Sanchez Dominguez, D., Frias, D., \& Marrero Iglesias, S. (2015). Using a Multi-Agent System for Monitoring Indicators of Quality of Service in Power Distribution Networks. IEEE Latin America Transactions, 13(4), 1048-1054. https://doi.org/10.1109/TLA.2015.7106356

Washington, U. (2018). Power Systems Test Case Archive-UWEE [Academic]. Power Systems Test Case. http://www2.ee.washington.edu/research/pstca/

Zimmerman, R. D., Murillo-Sanchez, C. E., \& Thomas, R. J. (2011). MATPOWER: Steady-State Operations, Planning, and Analysis Tools for Power Systems Research and Education. IEEE Transactions on Power Systems, 26(1), 12-19. https://doi.org/10.1109/TPWRS.2010.2051168 